\title{
Os desafios dos sistemas de informação em enfermagem: uma revisão narrativa da literatura
}

\author{
The challenges of nursing information systems: \\ a narrative review of the literature
}

Tiago Nascimento (https://orcid.org/0000-0003-3646-9057) ${ }^{1}$

Inês Frade (https://orcid.org/0000-0002-0590-4290) ${ }^{1}$

Susana Miguel (https://orcid.org/0000-0001-8830-070X) ${ }^{2}$

Maria Helena Presado (https://orcid.org/0000-0002-6852-7875) ${ }^{1}$

Mário Cardoso (https://orcid.org/0000-0003-4961-9026) ${ }^{1}$

${ }^{1}$ Escola Superior de Enfermagem de Lisboa. Av. Prof. Egas Moniz. 1600-096 Lisboa Portugal. tnascimento@esel.pt

${ }^{2}$ Instituto Português de Oncologia de Lisboa Francisco Gentil. Lisboa Portugal.

\begin{abstract}
Nursing information systems, where quality indicators are integrated, focus on the standardization of health records and the consequent visibility of the provided care. Despite the acknowledged importance of the contributions of information systems, their implementation has been characterized by several challenges, so we propose to reflect on them. To identify the evidence available in the literature on these same challenges, a narrative review of the literature was developed, with the analysis of relevant articles and reports on this issue. It is clear in the literature the importance of information systems for obtaining quality indicators that are sensitive to nursing care, with a positive impact on the quality of care, allowing for measurable quality in interventions, as well as facilitating inter and intra-institutional comparability, in real-time or in a retrospective analysis. The challenges encountered and which urgently needs to be resolved in clinical practice are related to the difficulty for professionals to perceive the impact of computer records, the visibility of nursing indicators and the time that is allocated in the context of providing care to carry out these records.
\end{abstract}

Key words Professional practice, Information systems, Quality indicators, Health care
Resumo Os sistemas de informação em enfermagem, onde se integram os indicadores de qualidade, têm como foco a uniformização dos registos em saúde e a consequente visibilidade dos cuidados prestados. Apesar da reconhecida importância dos contributos dos sistemas de informação, a sua implementação tem-se pautado por vários desafios pelo que nos propomos assim a refletir sobre estes. Com o objetivo de identificar a evidência disponível na literatura sobre estes mesmos desafios, foi desenvolvida uma revisão narrativa da literatura com análise de artigos e relatórios pertinentes acerca desta questão. Está patente na literatura a importância dos sistemas de informação para a obtenção de indicadores de qualidade sensíveis aos cuidados de enfermagem, existindo efetivamente um impacto positivo na qualidade dos cuidados, permitindo a mensurabilidade da qualidade nas intervenções, bem como facilitando a comparabilidade intra e interinstitucional, em tempo real ou em análise retrospetiva. Os desafios encontradose que urge resolver na prática clínica, relacionam-se com a dificuldade de os profissionais percecionarem o impacto dos registos informáticos, a visibilidade dos indicadores em enfermagem e o tempo que é alocado em contexto de prestação de cuidados para realizar estes registos.

Palavras-chave Prática profissional, Sistemas de informação, Indicadores de qualidade em assistência à saúde 


\section{Introdução}

Os sistemas de informação têm-se revelado uma mais valia na sistematização da informação, permitindo a salvaguarda de dados sensíveis e garantindo a interoperabilidade entre os vários atores do sistema de saúde.

No âmbito da enfermagem, foram criados com o pressuposto de extrair dados que permitissem o cálculo de indicadores sensíveis aos cuidados de enfermagem e, como consequência, um aumento da visibilidade dos cuidados, da valorização profissional e da monitorização da atividade.

Segundo a Comissão Europeia, a maioria dos países da Europa tem estratégias de medição de desempenho que visam melhorar a qualidade dos serviços de saúde. Essas estratégias normalmente incluem conjuntos de indicadores que são medidos ao longo do tempo; o número de indicadores varia entre menos de 30 (Áustria) e mais de 1.000 (Finlândia).

Os sistemas de informação podem ser compreendidos como um conjunto de procedimentos que pretendem transmitir informações entre indivíduos e órgãos através de qualquer meio ${ }^{1}$, são "a aplicação de uma perspectiva de sistemas totais na ligação de princípios teóricos relevantes com metodologias práticas para a administração eficaz das tecnologias da informação e suas aplicações para melhorar a prestação de serviços de saúde no contexto de ambientes de cuidados de saúde atuais e futuros"2.

Alguns dos sistemas de informação, como o SONHO, o SINUS e o cartão de utente têm-se revelado como desajustados, do ponto de vista funcional e tecnológico, apresentando diversas fragilidades ${ }^{3}$, pelo que nos surgiu a seguinte questão: quais os contributos e os desafios dos sistemas de informação para os indicadores de qualidade na prática clínica de enfermagem? Subjacente a esta questão surgiram as seguintes: de que forma os sistemas de informação podem promover a prestação segura de cuidados de enfermagem? De que forma poderão ser aproveitados os indicadores produzidos com recurso aos sistemas de informação para mudanças na gestão operacional? Que estratégias poderão ser desenvolvidas para dar visibilidade aos indicadores produzidos pelos enfermeiros no contexto de prestação de cuidados? Também na literatura é identificada esta lacuna, que para aumentar o uso dos sistemas, é necessário realizar outros estudos sobre os fatores que têm influência no aumento da motivação e interesses dos enfermeiros para usá-los ${ }^{4}$. Obje- tiva-se, assim, refletir sobre os desafios e o efetivo contributo dos sistemas de informação, integrando-os na prática dos profissionais e na prática baseada na evidência destes, dando sequência ao explorado por Nascimento 5 .

\section{Métodos}

Recorreu-se à metodologia de revisão narrativa da literatura ${ }^{6}$, com o objetivo de identificar os desafios dos sistemas de informação para os indicadores de qualidade na prática clínica de enfermagem, de forma a sustentar e a corroborar a reflexão sobre a temática em questão e dar resposta à questão norteadora: quais os contributos e os desafios dos sistemas de informação para os indicadores de qualidade na prática clínica de enfermagem? Foi realizada uma pesquisa no período de 5 a 25 de janeiro de 2020, nas bases de dados Medline (Medical Literature Analysis and Retrieval System Online Complete) e CINAHL ${ }^{\circ}$ (Cumulative Index to Nursing and Allied Health Literature Complete), utilizando os seguintes termos de pesquisa em inglês: information systems; nurs ${ }^{*}$; quality indicators.

Não se recorreu a definição de limites temporais por se considerar pertinente analisar a evolução das necessidades desta temática no decorrer do tempo. Como critérios de inclusão consideraram-se artigos nas línguas compreendidas pelos investigadores: português, inglês e espanhol bem como artigos com texto completo disponível. Como critérios de exclusão os artigos cujo título não contemplasse a temática de enfermagem. Para além das bases de dados foi realizada concomitantemente uma pesquisa livre recorrendo à plataforma Google Scholar, bem como pesquisas manuais das referências da literatura selecionada.

Obtiveram-se 119 artigos, que foram submetidos à aplicação dos critérios de inclusão e exclusão, resultando em 33 artigos. Após a leitura dos títulos e análise dos resumos foram selecionados 13 artigos aos quais se adicionaram 7 decorrentes das pesquisas manuais.

\section{Resultados e discussão}

Relativamente aos artigos analisados, fica patente que esta discussão se iniciou há cerca de 25 anos atrás, concomitante com a publicação de artigos a definir a problemática, o que é expectável tendo em conta a evolução dos sistemas informáticos bem como o acesso nos contextos, pelos Enfer- 
meiros. No entanto, o parco número de artigos encontrados revela que não existe ainda uma discussão abrangente e contínua, existindo sim artigos que vão demonstrando o trabalho realizado em contextos muito específicos, mas com poucos exemplos de integração transversal.

É inegável a importância dos sistemas de informação como uma importante ferramenta de trabalho e avaliação de qualidade, com uma mudança na perspectiva crescente em resultados e inteligência organizacional. Assumem ainda uma especial importância na medida em que devem contribuir para também medir o desempenho em saúde 7 .

No que respeita aos sistemas de informação em enfermagem, a Ordem dos Enfermeiros $(\mathrm{OE})^{8}$ alerta para a relevância, não só "dos imperativos de natureza legal e ética dos sistemas de informação como dos que decorrem da sua importância para as decisões clínicas, a continuidade e a qualidade dos cuidados, a gestão, a formação, a investigação e para os processos de tomada de decisão."

Considerando os sistemas de informação, estes contemplam 6 componentes (Figura 1).

Este autor considera assim os indicadores de qualidade como uma componente através da qual se conseguem retirar os dados que são essenciais para tomar decisões, sejam elas operacionais, táticas ou estratégicas. Para avaliarmos a

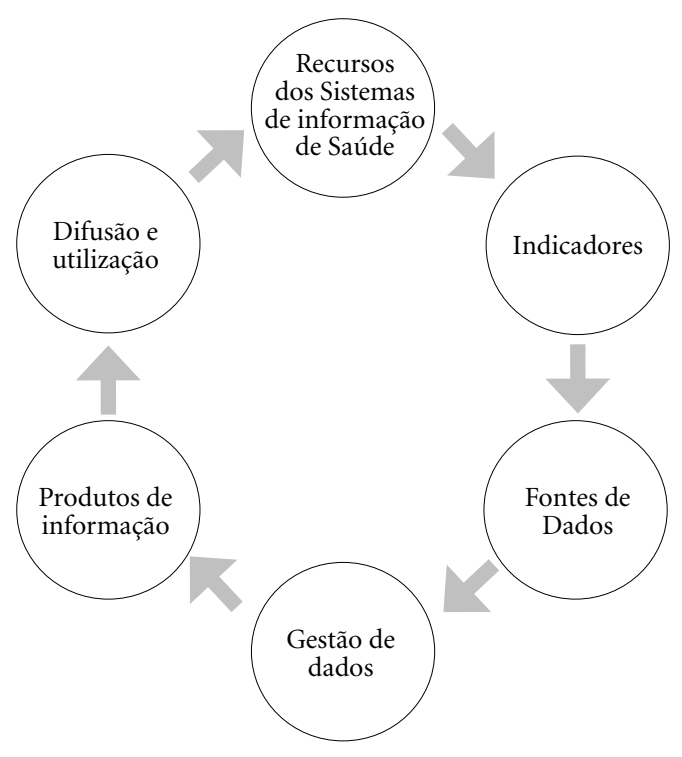

Figura 1. Componentes dos Sistemas de Informação9. qualidade, necessitamos que esta seja mensurável $^{9,10}$. As políticas em saúde de hoje têm por base um financiamento que assenta numa vertente maioritariamente médica, não considerando os cuidados prestados pelos enfermeiros na obtenção dos ganhos em saúde para a população.

A ausência da aplicação de indicadores é assim um handicap que tem tardado em ser colmatado. Segundo Pinto e Ferreira ${ }^{11}$, os "indicadores são ferramentas de gestão usadas para medir, monitorizar e avaliar os resultados de um processo, projeto ou política, a partir de dados registados de forma organizada e estruturada como parte dos sistemas de informação". Naqueles que são os documentos estruturantes da profissão, o regulamento de competências do enfermeiro de cuidados gerais, define, no capítulo C.2 - Melhoria da qualidade, ponto 89 , que o enfermeiro "utiliza indicadores válidos na avaliação da qualidade da prática de enfermagem”, tal como o ponto 90, em que o enfermeiro também "participa em programas de melhoria da qualidade e procedimentos de garantia da qualidade"8.

Os ganhos sensíveis aos cuidados de enfermagem definem-se como as evoluções positivas ou modificações operadas no estado dos diagnósticos de enfermagem, após as intervenções ${ }^{7}$, logo a mensurabilidade desse ganho é traduzida através de um indicador ${ }^{12}$. Esta mensurabilidade confirma-se, ainda no mesmo documento, no sentido em que a avaliação, quantitativa ou qualitativa, dos cuidados de enfermagem requer um conjunto de indicadores e unidades de medida que se verifiquem sensíveis aos mesmos e, por isto, diferentes, na maior parte dos casos, dos habitualmente utilizados noutras disciplinas ${ }^{7,13}$.

Assim, admiindo que o método mais razoável para a tomada de decisão a nível operacional é a monitorização longitudinal da produtividade dos cuidados de enfermagem nas unidades, combinado com indicadores de qualidade de cuidados aos utentes, a OE defende um modelo global para a avaliação da qualidade e da produtividade na enfermagem que contemple, concomitantemente, indicadores para a estrutura, para o processo e para o resultado ${ }^{8}$.

No entanto, para que estes indicadores encontrem expressão, é essencial que sejam registados, ou seja, a informação da intervenção realizada pelo Enfermeiro deve estar corretamente registada, não só no que respeita ao local, mas também em relação ao seu conteúdo. Este deverá ser o mais uniforme possível, permitindo que em qualquer região do país, em qualquer contexto de cuidados, Enfermeiros diferentes possam registar 
as mesmas intervenções da mesma forma, garantindo assim uma adequação e, acima de tudo, uma maior expressão no que diz respeito ao impacto. Se o registro de todo o processo de enfermagem prestado, não for efetuado, não se produzem indicadores de avaliação da qualidade. Isto traduz não só uma não visibilidade à sociedade da atuação da classe profissional em causa, como dificilmente transparece o processo de melhoria da qualidade dos cuidados de enfermagem ${ }^{14}$.

Devido à complexidade de um serviço de saúde, um único indicador não é capaz de avaliar a qualidade do serviço prestado aos clientes, sendo necessário elencar um grupo de indicadores de cada categoria para este fim ${ }^{15-17}$ ou pelo menos um indicador de cada categoria. A utilização da tecnologia permite a criação de indicadores informatizados que posteriormente irão proporcionar aos enfermeiros ferramentas de trabalho que permitem medir indicadores e desenvolver bases de dados de fácil gestão, contribuindo para melhoria contínua da qualidade dos cuidados ${ }^{11,18,19}$.

Existe um consenso mundial no sentido de que as evidências científicas demonstram cada vez mais a necessidade de implementar resultados que avaliem a contribuição dos cuidados à qualidade e à segurança clínica, destacando a existência de indicadores sensíveis à prática de enfermagem ${ }^{20}$. Estudos estimam que o uso concomitante de suporte informático e linguagem padronizadas fornecem informações completas e precisas, contribuindo para a qualidade dos registos de enfermagem ${ }^{11}$. As mesmas autoras sustentam 2 questões preponderantes, em primeira instância, que a qualidade dos cuidados está diretamente relacionada com a qualidade dos registros de enfermagem e, em segundo lugar, que o uso de linguagem padronizada combinada com as ferramentas informáticas contribui para uma melhor tomada de decisão.

As conceções dos enfermeiros destacaram que se trata de um sistema facilitador da prática, tendo em vista o acesso, a disponibilidade das informações, a rapidez, a praticidade, a clareza e a otimização do espaço físico ${ }^{21,22}$. Empiricamente os enfermeiros compreendem a importância dos sistemas de informação, na perspetiva da otimização dos recursos, bem como na facilidade no acesso e no aumento da segurança da informação registada.

Encontramo-nos numa fase de redução de recursos humanos, redução de orçamentos, aumento da atividade, da complexidade e, com a existência de novas tecnologias, é essencial que os serviços compreendam como prestam cuidados e que determinem de que forma estes são efetivos e eficientes ${ }^{23}$. Esta realidade corrobora a atual realidade portuguesa, apesar de mais de uma década de diferença.

Desta forma, é necessário que os enfermeiros sejam responsabilizados, por mais do que as suas intervenções, pelos resultados destas permitindo assim que exista uma maior preocupação com seu desempenho. Na mesma perspetiva, Akachi e Kurk ${ }^{24}$ definem que os sistemas de informação em saúde fornecem dados incompletos e, muitas vezes, não confiáveis, com muitos indicadores de utilidade incerta. Consideramos que as métricas existentes podem não ter a sensibilidade para traduzir o processo de cuidados e a experiência dos utentes no sistema de saúde.

Os resultados dos utentes que são sensíveis às práticas de cuidados de saúde, são um pilar de avaliação de qualidade e raramente recolhidos. Estes autores ${ }^{24}$, propõem seis políticas para melhorar a qualidade da mensurabilidade do atendimento e amplificar seu impacto político: (i) redobrar esforços para melhorar e institucionalizar o registo dos sinais vitais; (ii) realizar pesquisas sobre instalações de reforma e fortalecer os sistemas de informação; (iii) promover novas medidas de qualidade para contextos de baixos recursos; (iv) obter a perspetiva do utente acerca da qualidade dos cuidados; (v) investir em dados nacionais de qualidade; e (vi) traduzir a qualidade em evidência com impacto para a política.

Os desafios mais importantes no uso dos sistemas de informação são os fatores relacionados ao ambiente humano e aos fatores humanos, pelo que é indissociável a envolvência dos profissionais, conscientes e capacitados, que trará como reflexo um trabalho bem estruturado, planeado e organizado, com influência direta na atenção integral ao cliente, nos processos de melhoria dos sistemas de informação ${ }^{4,22}$.

Além desta perspectiva dos profissionais, é também essencial que os sistemas permitam uma adequada transmissão de informação entre si, garantido a informação ao profissional de saúde sobre cada cliente, em tempo real, garantido a segurança deste em todo o momento, mas evitando assim repetições e perdas de informação ${ }^{25,26}$.

\section{Considerações finais}

A literatura corrobora aquelas que são as reflexões empiricamente realizadas numa perspectiva em que os sistemas de informação se configuram como o futuro para a visibilidade e a avaliação de 
desempenho dos cuidados prestados. No entanto, necessita de ser consolidado no que concerne à translação dos resultados para a prática e com impacto nas políticas de saúde.

É essencial que se desenvolvam mecanismos que permitam uma maior interoperabilidade entre os vários sistemas, aumentando em simultâneo a capacidade de serem user friendly, auscultando os profissionais de saúde dos vários níveis de cuidados.

No que respeita às limitações metodológicas do estudo, a literatura existente ainda não permite uma análise mais aprofundada da temática, sendo por isso, relevante desenvolver investigação que colmate esta lacuna na evidência científica, com enfoque na perceção dos profissionais ao longo dos anos e tendo em conta as limitações dos recursos à disposição, bem como, de que forma estes registos podem expressar a maior dependência dos utentes, mas também de que forma retiram horas de cuidados necessárias a estes.

É assim relevante o reconhecimento desta lacuna existente, o que permite sugerir futuras investigações neste âmbito, com vista ao aprofundamento do fenómeno em análise e consequente melhoria da prática instituída.

\section{Colaboradores}

T Nascimento e I Frade: desenho e elaboração do artigo; análise e interpretação dos dados; redação do manuscrito. S Miguel: análise e interpretação dos dados; revisão de versões e revisão crítica do conteúdo. MH Presado e M Cardoso: revisão de versões e revisão crítica do conteúdo. 


\section{Referências}

1. Benito GAV, Licheski AP. Sistemas de Informação apoiando a gestão do trabalho em saúde Sistemas de Informação apoiando a gestão do trabalho em saúde. Reben 2009; 62(3):447-450.

2. Tan JKH. Health Management Information Systems: Theories, Methods, and Applications. New York: Aspen Publishers Inc.; 1995.

3. Espanha R. Sistemas de Informação em Saúde e Saúde Online. Tecnologias de Informação e Comunicação Adenda 2010; 1-12.

4. Ahmadian L, Dorosti N, Khajouei R, Hajesmaeel Gohari S. Challenges of using Hospital Information Systems by nurses: comparing academic and non-academic hospitals. Electron Physician 2017; 9(6):46254630.

5. Nascimento T, Frade I, Miguel S, Presado H, Cardoso M. Os sistemas de informação em enfermagem e os indicadores de qualidade: contributos e desafios para a prática clínica. In: Atas - Investigação Qualitativa em Educação CIAIQ2019; 2019; Lisboa. 1:965-970.

6. Green B, Johnson C, Adams A. Writing a narrative literature reviews for peer-reviewed journals: secrets of the trade. J Chiropr Med 2006; 5(3):1-13.

7. Fernandes S, Tareco E. Sistemas de informação como indicadores de qualidade na saúde. Uma revisão de níveis de abordagem. Risti 2016; 19:34-45.

8. Ordem dos Enfermeiros. Resumo Mínimo de Dados e Core de Indicadores de Enfermagem para o Repositório Central de Dados da Saúde. Sist Informação Enferm [Internet]. 2007; 16. [cited 2019 Apr 16]. Available from: http://www.ordemenfermeiros.pt/ documentosoficiais/documents/rmde_indicadoresvfout2007.pdf

9. Sandi A. A importância dos Sistemas de Informação em Saúde - Estudo de caso na USF CelaSaúde. Coimbra: FEUC; 2015.

10. Weiner J, Balijepally V, Tanniru M. Integrating Strategic and Operational Decision Making Using DataDriven Dashboards: The Case of St. Joseph Mercy Oakland Hospital. J Health Manag 2015; 60(5):319330.

11. Pinto VRS, Ferreira SCM. Computerized tool to calculate nursing quality indicators: a methodological research. Online Brazilian J Nurs 2014; 13:382-385.

12. Harris C, Allen K, Ramsey W, King R, Green S. Sustainability in Health care by Allocating Resources Effectively (SHARE) 11: Reporting outcomes of an evidence-driven approach to disinvestment in a local healthcare setting. BMC Health Serv Res. 2018; 18(1):386.

13. Ordem dos Enfermeiros (OE). Sistema de Informação de Enfermagem (SIE) Princípios básicos da arquitectura e principais requisitos técnico - funcionais. Lisboa: $\mathrm{OE}$; 2007.

14. Liang Y-W, Chang H-F, Lin Y-H. Effects of health-information-based diabetes shared care program participation on preventable hospitalizations in Taiwan. BMC Health Serv Res 2019; 19(1):890.

15. Sharma A, Rana SK, Prinja S, Kumar R. Quality of Health Management Information System for Maternal \& Child Health Care in Haryana State, India. PLoS One 2016; 11(2):e0148449.
16. Malik AM, Schiesari LMC. Qualidade na Gestão Local de Serviços e Ações de Saúde. Série Saúde e Cid 1998; 241.

17. Lima CSP, Barbosa SFF. Patient Safety in Critical Care Unit: Development of a Nursing Quality Indicator System. Stud Health Technol Inform 2015; 216:251254.

18. Báo ACP, Amestoy SC, Moura GMSS, Trindade LL. Indicadores de qualidade: ferramentas para o gerenciamento de boas práticas em saúde. Rev Bras Enferm 2019; 72(2):377-384.

19. Southern DA, Hall M, White DE, Romano PS, Sundararajan V, Droesler SE, Pincus HA, Ghali WA. Opportunities and challenges for quality and safety applications in ICD-11: an international survey of users of coded health data. Int J Qual Heal Care 2016; 28(1):129-135.

20. Porcel-Gálvez AM. Resultados sensíveis à prática de enfermagem: pesquisa clínica e evidência. Rev Gaúcha Enferm 2019; 40:e20190316.

21. Westra BL, Latimer GE, Matney SA, Park JI, Sensmeier J, Simpson RL, Swanson MJ, Warren JJ, Delaney CW. A national action plan for sharable and comparable nursing data to support practice and translational research for transforming health care. J Am Med Informatics Assoc 2015; 22(3):600-607.

22. Lima DFB, Braga ALS, Fernandes JL, Brandão ES. Sistema de informação em saúde: conceções e perspetivas dos enfermeiros sobre o prontuário eletrónico do paciente. Revista de Enfermagem Referência 2011; Vol. serIII:113-119.

23. Long LE, Mha RGN. Imbedding quality improvement into all aspects of nursing practice. Int J Nurs Pract 2003; 9(5):280-284.

24. Akachi Y, Kruk ME. Quality of care: measuring a neglected driver of improved health. Bulletin of the World Health Organization 2017; 95:465-472.

25. Mota L, Pereira F, Sousa P. Sistemas de Informação de Enfermagem: exploração da informação partilhada com os médicos. Rev Enf Ref 2014; IVSérie(1):85-91.

26. Labbadia LL, D'Innocenzo M, Fogliano RRF, Silva GEF, Queiroz RMRM, Carmagnani MIS, Salvador ME. Sistema informatizado para gerenciamento de indicadores da assistência de enfermagem do hospital São Paulo. Rev da Esc Enferm 2011; 45(4):1013-1017.

Artigo apresentado em 27/04/2020

Aprovado em 04/06/2020

Versão final apresentada em 06/06/2020

Editores chefes: Maria Cecília de Souza Minayo, Romeu Gomes, Antônio Augusto Moura da Silva 